\title{
Applications of Micellar Liquid Chromatography in Bioanalysis: A Review
}

\author{
Kalyankar T. M. , Kulkarni P.D., Wadher S. J. and Pekamwar S. S. \\ Department of pharmaceutical chemistry, School of Pharmacy, Swami Ramanand Teerth Marathwada University, Nanded-431606, Maharashtra, India.
}

\begin{tabular}{|c|c|}
\hline ARTICLE INFO & ABSTRACT \\
\hline Article history: & \multirow{8}{*}{$\begin{array}{l}\text { Micellar liquid chromatography (MLC) is one of the very important analytical techniques. It contains mobile } \\
\text { phase added with surfactants above its critical micellar concentration and the stationary phase is modified with } \\
\text { surfactant monomers. So, micelles alter the solubilising capability of the mobile phase which forms diverse } \\
\text { interactions with major implications in retention and selectivity. It is an alternative to conventional reversed } \\
\text { phase liquid chromatography. It allows direct injection of physiological fluids, analysis of pharmaceutical } \\
\text { compounds, physiological partitioning process. MLC (micelle liquid chromatography) has proved time saving as } \\
\text { compared to other analytical technique like HPLC and ion-pairing. Applicability of MLC is increased in the field } \\
\text { of bioanalysis. It is used to analyze different samples of drugs in serum, urine, food etc. In this review we have } \\
\text { focused on the various examples of use of MLC in bioanalysis. This review also contains basic information about } \\
\text { MLC such as micellar mobile phase, stationary phase, surfactants, and fundamental studies such as retention } \\
\text { behavior and partition coefficient. }\end{array}$} \\
\hline Received on: 30/07/2013 & \\
\hline Revised on: 25/12/2013 & \\
\hline Accepted on: 09/01/2014 & \\
\hline Available online: $30 / 01 / 2014$ & \\
\hline Key words: & \\
\hline MLC, bioanalysis, micelles, & \\
\hline $\begin{array}{l}\text { partition coefficient and } \\
\text { retention behavior. }\end{array}$ & \\
\hline
\end{tabular}

\section{INTRODUCTION}

MLC is the alternative to conventional reversed phase liquid chromatography in which surfactants are added to the mobile phase above its critical micellar concentration (CMC). It confers analytical procedures with greater accuracy and at lower cost. MLC is versatile in nature because of the various interactions established among the eluted solutes, the aqueous phase, the stationary phase, and micelles. MLC allows analysis of various compounds. The benefits of the addition of surfactant in mobile phase include modification of the interactions established inside the column, reduction in the quantity of organic solvent in mobile phase because of low evaporation, accuracy etc. MLC is a type of reversed-phase liquid chromatographic (RPLC) systems, in which stationary phase is a non-polar and mobile phase is a polar aqueous. However in conventional RPLC hydro-organic mobile phase is homo-geneous, but in MLC it is heterogeneous. It is composed of two different media: the micellar aggregates i.e.

* Corresponding Author

Dr. Kalyankar T. M., School of Pharmacy,Swami Ramanand Teerth Marathwada University, Nanded -431606; India.

E-mail:drkalyankartm@gmail.com micellar pseudophase and the aqueous-organic solvent containng surfactant monomers concentration equal to the critical micellar concentration (CMC). Surfactant monomers adsorb on the stationary phase, and create a structure like an open micelle, which reduce silanophilic interactions. The nonionic surfactants cause change only in the polarity of the stationary phase. But in case of ionic surfactants, positive or negative charge appear on its surface with major implications.

Recently a new micellar chromatographic mode has been described: high submicellar chromatography (Maria,2012; RuizAngel, 2008) in which the organic solvent content is high and surfactant forms micelles. This mode complements low submicellar chromatography and incresases number of applications of this chromatographic technique. Fundamental studies in MLC have not only served to develop the technique but also to establish its theoretical basis which enables its use in diverse applications.

\section{Basics of MLC}

Surfactants, micellar mobile phase and stationary phase are the basic and very important parts of MLC. In this section we have focused on each of them. 
Table. 1: Represents various types of surfactants often used in the micellar liquid chromatography.

\begin{tabular}{|c|c|c|c|c|c|c|}
\hline Compound & Name & Abbr. & $\begin{array}{c}\text { CMC } \\
\mathbf{m m}\end{array}$ & $\begin{array}{c}\text { Aggregation } \\
\text { No } \\
\end{array}$ & Kraft point & $\begin{array}{c}\text { Molar volume } \\
\text { Mol/L }\end{array}$ \\
\hline $\mathrm{C}_{12} \mathrm{H}_{25} \mathrm{SO}_{4} \mathrm{Na}$ & Sodium dodecyl sulfate & SDS & 8.1 & 62 & 16 & 0.246 \\
\hline $\mathrm{C}_{16} \mathrm{H}_{33} \mathrm{~N}\left(\mathrm{CH}_{3}\right)_{3} \mathrm{Br}$ & Cetyltrimethyl ammonium bromide & СТАB & 0.83 & 90 & 26 & 0.364 \\
\hline $\mathrm{C} 12 \mathrm{H} 25(\mathrm{C} 2 \mathrm{H} 4 \mathrm{O})_{23} \mathrm{OH}$ & Polyoxyethylene 23 dodecyl ether & Brij35 & 0.06 & 41 & $100>$ & 1.12 \\
\hline $\mathrm{C}_{14} \mathrm{H}_{22} \mathrm{O}\left(\mathrm{C}_{2} \mathrm{H}_{4} \mathrm{O}\right)_{9.5}$ & P-Octyl benzene polyoxyethylene 9.5 alcohol & Triton $\mathrm{x}-100$ & 0.3 & 140 & 67 & 0.743 \\
\hline
\end{tabular}

\section{Surfactants used in MLC}

Surfactant at low concentration has the property to adsorb onto the surfaces or interfaces of the system and alter the surface or interfacial free energies upto a marked degree (Rosen, 2004).

Surfactant posses both hydrophilic and hydrophobic parts. So, they have very interesting property of self organization in solution (David, 2009; Texter,1999). The hydrophobic nature is given by the tail of the molecule whereas the hydrophilic nature is provided by the head. Surfactants are classified into various classes viz. Anionic, cationic, nonionic, and amphoteric. The classification depends upon the charge of the hydrophobic head group.

The anionic surfactant dissociates in aqueous solutions and gives a negatively charged surface active portion whereas, the cationic surfactant dissociates in an aqueous solution and gives positively charged surface active portion. Nonionic surfactants contain polar group like an ether, carbonyl, amino group or alcohol as hydrophilic portion. Zwitter ionic surfactants have both positive and negative charges on the same molecule (Berthod and Garcia 2000, Myers 1988). The choice of appropriate surfactants depends upon various properties like CMC, krafft point, cloud point etc. Table no 1 gives the most oftenly used surfactants in MLC.

\section{Critical Micellar Concentration}

A surfactant with low CMC is suitable for MLC. Surfactants with high CMC value form a viscous solution and give undesirable high system pressure as well as background noise in UV detectors. The CMC values are low for Sodium dodecyl sulfate (SDS), Cetyltrimethyl ammonium bromide (CTAB), Brij 35. So, these surfactants are very commonly used in MLC. The organic solvents influence CMC values of surfactants and reduce the retention in MLC (Lopez 1998). New ionic liquid-based surfactants are also introduced eg.1-hexadecyl-3-butylimidazolium bromide which are suitable for MLC (Pino et al., 2009, Galgano and Seoud 2010).

\section{Krafft Point}

It is the temperature at which the solubility of an ionic surfactant monomer is equal to the CMC (Murray and Hartley 1935). There are no micelles below Krafft point. The chromatographic work in MLC should be conduted above Krafft point to avoid surfactant precipitation, which suggests that the Krafft point of a surfactant should be lower than room temperature. The Krafft point for SDS is $15^{\circ} \mathrm{C}$ whereas for CTAB it is around $20-25^{\circ} \mathrm{C}$ (Sehgal and Otzen, 2006; Beyer et al., 2006).

\section{Cloud Point}

In case of nonionic surfactants, if specific temperature is exceed, phase separation may occur. It is called as Cloud Point (Borgerding and W. L. Hinze,1985). So the work with these surfactants should be carefully conducted below the Cloud Point. Eg. for aqueous 1-6\% solutions of Brij-35 the cloud point is $100^{\circ} \mathrm{C}$ whereas for Triton $\mathrm{X}-100$ this value is $64^{\circ} \mathrm{C}$.

\section{Micellar mobile phase}

As mentioned above micellar mobile phase is one of the very important part of MLC. Micellar mobile phase contains surfactants above its critical micellar concentration. Above the CMC, a change in surfactant concentration is translated into an increase in the concentration of micelles in the solution, whereas the number of monomers of surfactant in the mobile phase remains constant.These micelles have three sites of solubilisation -the core which is hydrophobic in nature, the surface which is hydrophilic in nature, and the palisade layer i.e. the region between the core and the surfactant head groups. In the micelles there is a microenvironment which is different from that of bulk solvent and solutes associated with these micelles experience this environment (Delgado et al., 2004). Mostly separations in MLC are carried out with hybrid micellar mobile phases which is a buffered medium that contains micelles, molecules of organic solvent, surfactant monomers and water. The presence of organic solvent alters the micelle structure by reducing the polarity of the aqueous solution. The separation mode is predominantly micellar in nature, which is disturbed by the organic solvent. So there may be a change in parameters, such as surfactant aggregation number and the CMC . The type of organic solvent and surfactant decides maximal allowable concentration of organic solvent.

\section{pH of the Mobile Phase}

The packing materials for MLC is same as classical RPLC, having a limited working $\mathrm{pH}$ range of 2.5-7.5. Suitable $\mathrm{pH}$ values for chromatographic work depend on analytes and the surfactant selected. Phosphoric or Citric acid buffers are used to fix the $\mathrm{pH}$ of the micellar mobile phase. Potassium salts are not recommended for mobile phases containing SDS as potassium dodecyl sulfate have a high Krafft point which results in precipitation from aqueous solutions (Berthod and Garcia, 2000).

\section{Organic Solvents}

The polarities of the analytes decide which organic solvent modifier should be used for MLC. For polar analytes, 1propanol, 2-propanol, or acetonitrile are used for short retention times (below $20 \mathrm{~min}$ ), whereas for nonpolar compound stronger 
solvents such as 1-butanol or 1-pentanol are needed. These organic solvents can also used for those compounds which shows high affinity for the surfactant adsorbed on the stationary phase (RuizAngel et al., 2002). The two latter alcohols i.e. 2-propanol, or acetonitrile give rise to micro emulsion formation at sufficiently high concentrations (Bryant and Altria, 2004). The lower organic solvent consumption reduces cost and toxicity, which helps for "green chemistry". Organic solvent in the micellar media decreases evaporation of micellar mobile phases and thus it can be preserved for a long time period with no significant changes in the composition. However, quantity of the organic solvent that can be added is depends upon its solubility. High organic solvent concentration causes the disaggregation of micelles and the only free surfactant molecules remain in the mobile phase. The organic solvent contents (in percent) which maintain the integrity of micelles are $<15 \%$ for propanol and acetonitrile, $6 \%$ for pentanol , and $10 \%$ for butanol (Lopez-Grio et al., 2000). These values are quite less as compared with those needed in classical RPLC.

\section{MODIFIED STATIONARY PHASE}

\section{Surfactant Adsorption}

The most widely used stationary phase in MLC is alkylbonded C18 column, but other columns like C8 or cyanopropyl may be selected. SDS, CTAB, Brij -35 these surfactants very strongly modifies alkyl-bonded phase when incorporated into the mobile phase. They adsorb on the porous RPLC packing and drastically affect the chromatographic retention, which leads to change in various surface properties of the stationary phase like structure, polarity, surface area and pore volume. The surfactant molecules also reduce the volume of stationary phase pores (Borgerding et al.,,1989). The addition of ionic compounds in micellar mobile phase gives ionic strength. Salt addition changes the amount of adsorbed ionic surfactant because there is a reduction in both electrostatic repulsion and surfactant CMC (Berthod et al., 1986).

Surfactants coat the bonded-stationary phase and thus full similar coating would make the stationary phases all similar. According to solid-state NMR studies of SDS, the hydrophobic tail of SDS associated to C18 alkyl-chain bonded with silica stationary phase whereas the sulfate head group is oriented away from the surface (Lavine et al.,, 1996). This gives rise to a negatively charged hydrophilic layer. Hence the penetration depth of solutes is affected into the bonded phase. In the case of CTAB with alkyl-bonded phases, surfactant adsorption leads to a more hydrophobic stationary phase because the cationic trimethylammonium head group is partially incorporated into the bonded phase, associated to free silanols .In contrast, on cyanobonded phases, both charged surfactants (SDS and CTAB) are adsorbed head down with their tails projected outwards, creating thus pseudo-alkyl bonded phases. The smaller surface charge of the modified cyano-bonded phases is responsible for the more important role of solute-micelle interactions for charged solutes.

\section{Interactions of surfactant monomer with stationary phase}

The interactions between phase surfactant monomer and stationary phase play important role in retention of solutes and make MLC more complex as compared to conventional RPLC (Armstrong, 1981).

\section{Hydrophobic interaction}

In which the alkyl tail of the surfactant monomer is in contact with the nonpolar ligand of the stationary phase and the ionic head group of surfactant monomer is directed away from it. In this way, the stationary phase got some ion exchange ability with charged solutes

\section{Silanophilic interaction}

Silanophilic interaction where the ionic head group of the surfactant would be adsorbed on the stationary phase. There is also a possibility of competition between surfactant and solute for the stationary phase (Armstrong, 1981). So, MLC is more complex than conventional RPLC with hydro - organic solvent giving rise to number of interactions like electrostatic, hydrophobic and steric (Heranadez, 1992). Micelles provide hydrophobic as well as electrostatic sites for interactions with solutes (Ruiz-Angel et al., 2009). This leads MLC to determine almost any compound.

\section{Partition Coefficient}

In MLC the chromatographic behavior is explained by considering three phases viz. stationary phase, micellar pseudophase and bulk solvent. Solutes get separated according to their differential partitioning between micelles and bulk solvent either in the mobile phase or in surfactant- coated stationary phase. Water-insoluble species get separated directly between the modified stationary phase and the micellar pseudophase. The partitioning behavior in MLC can be explained by three coefficients: $P_{\mathrm{sw}}$ (the partition between aqueous solvent and stationary phase), $P_{m w}$ (between aqueous solvent and micelles), and $P_{\mathrm{ms}}$ (between micelles and stationary phase). The solute affinity with the stationary phase and micelles is denoted by the coefficients $P_{\mathrm{sw}}$ and $P_{\mathrm{mw}}$ respectively. These two coefficients have contradictory effects on solute retention: as the $P_{\mathrm{sw}}$ increases, the retention increases due to affinity with stationary phase. On the other hand, increase in $P_{\text {wm }}$ causes reduction in the retention due to the stronger association with micelles.

$$
\frac{V_{S}}{V_{e}-V_{m}}=\frac{\left(V\left(P_{M W}-1\right)\right.}{P_{S W}}[M]+\frac{1}{P_{S W}} \quad \ldots \ldots \text { (1) }
$$

Where-

$V e=$ retention volume of the solute,

$V s=$ The volume of the stationary phase,

$\mathrm{V}=$ The partial specific volume of the surfactant in the micelle,

$V m=$ The volume of the mobile phase,

$[\mathrm{M}]=$ The micellized surfactant concentration, i.e. (total surfactant concentration minus the $\mathrm{CMC}$ ) in moles per liter;

$P s w=$ The partition coefficients of the solute between the stationary phase and water, and $\mathrm{P} m w=$ The partition coefficient of solute between the micelle and water respectively. 
The interactions of the solutes with surfactant coated stationary phase and micelles explain the retention behavior. The elution of charged and neutral species is affected by dipole-dipole, nonpolar, and proton donor-acceptor interactions (Marina 1996). However, the charged solutes interact electrostatically with the ionic surfactants. Two conditions are possible with ionic surfactants accordingly the charge of surfactant and solute: repulsion or attraction.

If there is electrostatic repulsion between ionic surfactant and solute, the stationary phase does not retain the charged solutes and elute early at the dead volume, except only any hydrophobic reaction with the modified bonded layer exist there. On the other hand, electrostatic attraction and hydrophobic interactions with the stationary phase lead to strong retention in MLC. If appropriate surfactant is chosen the mixtures of polar and nonpolar solutes can also be resolved effectively.

\section{Retention Behavior}

Many theoretical approaches have been proposed to explain the retention behavior of binding solutes as a function of the "micellar" concentration $[M]$ ( which should be understood as the concentration of monomers of surfactant forming micelles, calculated as the total concentration of surfactant minus the CMC) (García, 1997).

The partitioning model of Armstrong and Nome (Armstrong, 1981), the equilibrium approaches of Arunyanart and Cline-Love (Arunyanart 1984), and Foley (Foley,1990) are important and they are proved as milestone in forming therotical basis for MLC. The proposed models allow measuring the strength of solute-micelle and solute-stationary phase interactions.

\section{Armstrong and Nome model}

This model assumes transitions among 3 environments $i$. e. water, micelles and stationary phase in micellar chromatographic system.

$$
\frac{V_{e}-V_{O}}{V_{S}}=\frac{K}{\phi}=\frac{P_{W S}}{1+v\left(P_{W M}-1\right)[M]} \ldots \ldots
$$

Where,

$\mathrm{Ve}=$ The total volume of mobile phase needed to elute a given solute from the column.

$V s=$ The volume of the active surface of the stationary phase,

$V 0=$ The column void volume,

$\square=\mathrm{Vs} / \mathrm{V} 0$ i.e. phase ratio

$V=$ Partial specific volume of monomers of surfactant in the micelle (Esteve-Romero, 2005)

\section{Arunyanart and Cline-Love model}

Arunyanart and Cline-Love considered association equilibria of solute in bulk aqueous solvent (A) with the stationary phase binding sites (S) and with monomers of surfactant in the micelle (M), governed by the binding constants $K_{\mathrm{AS}}$ and $K_{\mathrm{AM}}$, respectively.

$$
K=\phi \frac{[A S]}{[A]+[A M]}=\frac{\phi K_{A S}[S]}{1+K_{A M}[M]} \ldots \ldots
$$

\section{Foley model}

Foley put forward the idea of treating the retention factor. (Hoonka et al., 2012).

$$
K=K_{O} \frac{1}{1+K_{A M}[M]} \ldots \ldots(4)
$$

This model resembles previous two models as the retention factor of free solute $\left(\mathrm{k}_{0}\right)$ has coincided with $\mathrm{P}_{\mathrm{ws}}$ in Armstrong and Nome model model and $\mathrm{K}_{\mathrm{AS}}$ in Arunyanart and Cline-Love model whereas $\mathrm{K}_{\mathrm{AM}}$ coincide with $\mathrm{K}_{\mathrm{AM}}$ in Arunyanart and Cline-Love.

\section{MLC Applications In Bioanalytical Chemistry}

Micellar liquid chromatography is used for various applications. It has been used in the analysis a range of biological samples. It includes direct injection of physiological fluids, the analysis of pharmaceutical compounds. It also has major role in physiological partitioning processes (Srisom et al.,.2007; GilAgusti et al., 2007; Torres-Cartas et al., 2007; Martinez-Algaba et al., 2006; Rambla-Alegre et al., 2006; Kulikov et al.,,2005; Capella-Peiro 2005 ). MLC has proved time saving as compared to other analytical methods like HPLC and ion-pairing. Because it does not require sample extraction or any other sample preparation. The peak shapes obtained are superior too (Nishi,1997). The MLC reduces the excessive peak tailing of basic drugs which is very common in ion pairing whereas hydrophilic drugs are retained which are unretained in HPLC. Another very important application of MLC is the analysis of physiological samples. Micelles solubilize proteins which make possible MLC to analyze urine, serum, and plasma (Khaledi,1997). Here we are discussing some examples of use of MLC in bioanalytical chemistry.

\section{Analysis of biogenic amines in food samples}

Increased concentrations of biogenic amines in food cause toxicity. These compounds are hard to destroy by various processes like pasteurization and cooking. So, it is very important to control the production process. Micellar liquid chromatography is able to determine the biogenic amines from the food samples. Here we gave examples of some MLC methods which are used to determine these biogenic amines.

\section{Separation of Tryptamine and Tyramine from wine}

This method was developed to determine two biogenic amines viz. Tryptamine (TryptA) and Tyramine (TyrA), with their precursors, Tryptophan (TryptO) and Tyrosine (TyrO) respectively in wine by a liquid chromatographic procedure (Gil-Agusti et al., 2007). The mobile phase containing 0.15 M SDS and 5\% 1propanol having $\mathrm{pH} 3$ successfully resolved the four subsequent compounds with an analysis time of $16 \mathrm{~min}$. The column used was $\mathrm{C} 18$ with electrochemical detection. The potential selected was 0.8 $\mathrm{V}$ after checking the oxidation potential at $0.6-0.9 \mathrm{~V}$. Linearities were studied in the concentration range of $0.1-10 \mu \mathrm{g} / \mathrm{ml}$. The regression coefficient was $(r>0.996)$. The RSD values obtained were lower than 2.6 and $4.8 \%$, respectively. This procedure was tested in a number of types of wines and no matrix effect was 
observed . These results show that the direct sample introduction simplifies and greatly expedites these types of procedures, reducing their cost and improving their accuracy.

\section{Determination of Putrescine and Tyramine in Fish}

The MLC method was developed to determine two biogenic amines viz. Putrescine and Tyramine in fish samples (Mei-Liang et al., 2011). The fish samples were injected directly into a chromatograph, after derivatization with 3, 5-dinitrobenzoyl chloride without any previous extraction. The mobile phase selected contains $0.15 \mathrm{M}$ SDS-6\% $(v / v)$ and butanol at $\mathrm{pH} 7$. The column used was $\mathrm{C} 18$ with UV detection of derivatives set at 260 $\mathrm{nm}$. Linearities were studied in the range from 0.5 to $1000 \mathrm{ppm}$ with regression coefficient $(r>0.999)$. The results obtained for accuracy between $99.5 \%$ and $102.7 \%$ respectively. This method is suitable for the analysis of these compounds in fish sauces.

\section{Analysis of Anserine and carnosine in meat samples}

Natural antioxidants Anserine and Carnosine are present in some types of meat(Gil-Agusti et al., 2007). The method developed for these two peptides by using a micellar mobile phase of $0.1 \mathrm{M}$ sodium dodecyl sulphate of $\mathrm{pH} \mathrm{7,} \mathrm{an} \mathrm{amino} \mathrm{column} \mathrm{and}$ UV detection.

The analysis time was $12 \mathrm{~min}$. The regression coefficient obtained was $(r>0.998)$. The meat samples used were collected from poultry, pork and beef and directly injected into the chromatographic system. The direct injection of sample in micellar liquid chromatography overcomes many problems like chemical changes and losses in the analytes,toxicity,inflammation.

\section{Determination of Psychoactive Drugs}

Psychoactive drugs like Trazodone, Barbiturates, Benzodiazepines are successfully determined from serum samples, urine or blood samples by micellar liquid chromatograophy. These samples are chromatographed directly without any pretreatment like extraction .

\section{Trazodone (TRZ) in pharmaceuticals and in urine samples}

Trazodone (TRZ) is a second-generation non-tricyclic antidepressants drug. TRZ has been determined in pharmaceutical formulations and in urine samples [Carda-Broch et al., 2007]. For the analysis of TRZ in pharmaceuticals flurometric detection was carried out at $325 \mathrm{~nm}$ (excitation) and at $440 \mathrm{~nm}$ (emission). In this method the column used was $\mathrm{C} 18$ and mobile phase selected was $0.2 \mathrm{M}$ SDS with $8 \%$ butanol. The $\mathrm{pH}$ of the mobile phase was maintained to 3 with phosphate buffer. The solute eluted in 10 $\min$. The linearity was studied in the range of $0.3-5 \mu \mathrm{g} / \mathrm{ml}$, and the regression coefficient was $(r>0.999)$. The analysis of TRZ in urine does not require an extraction step.

The linearity ranges were $0.06-22.4 \mu \mathrm{g} / \mathrm{ml}$, showing regression coefficient was $(r>0.9991)$. This method showed good accuracy, repeatability, and selectivity. Repeatability and intermediate precision were studied for various concentrations of the drug, with good precision values $(<2 \%)$. The recoveries obtained were (93-110\%) from urine samples. In conclusion, this procedure is suitable for routine analyses of the drug.

\section{Analysis of less prescribed benzodiazepines}

Clotiazepam, Clozapine and Pinazepam are less prescribed but important benzodiazepines. The chromatographic method has been developed for Clotiazepam, clozapine and pinazepam in serum (Hoonka et al., 2012). The serum samples were injected directly, without any pretreatment. In the present method the conditions maintained for the analysis of the three benzodiazepines were mobile phase containing 0.13 M SDS with $2.4 \%$ pentanol, $0.01 \mathrm{M}$ phosphate buffer, $0.1 \%$ triethylamine at $\mathrm{pH}$ 7. Temperature maintained to $25^{\circ} \mathrm{C}$. UV detection was carried out at $240 \mathrm{~nm}$ and column used was $\mathrm{C} 8$. The elution time was less than $8 \mathrm{~min}$.

\section{Determination of some barbiturates in serum}

A procedure was developed for the determination of several barbiturates viz. Amobarbital, Barbital, Hexobarbital and Secobarbital, using MLC and C18 column (120 x 4.6mm). The mobile phase used was 0.1M SDS-4\% (v/v) butanol at pH 7 (Elisa et al., 2002). The four barbiturates were determined in the UV ranges at $230 \mathrm{~nm}$. In the proposed MLC procedure linearities were studied and regression coefficient obtained was ( $r$ >0.999). This method could be useful for recovery studies of barbiturates in serum sample that agreed with the concentration added.

\section{Determination of benzodiazepines in serum}

This MLC method is useful for the determination Diazepam, Bromazepam, Flunitrazepam, Oxazepam ,Halazepam, Medazepam, Tetrazepam and Nitrazepam (Capella-Peiro et al., 2002). In the proposed method, the mobile phase used was $0.06 \mathrm{M}$ SDS-5\% butanol-0.01 $\mathrm{M}$ phosphate buffer at $\mathrm{pH} 7$ and the temperature maintained to $25 \mathrm{C}$. The column used was $\mathrm{C} 18$. The detection of benzodiazepines was carried out in UV range at 230 $\mathrm{nm}$. The serum samples were injected directly. The MLC results were compared with those obtained from a conventional HPLC method using methanol-water 5:5 (v/v) which requires a previous extraction procedure.

\section{Analysis of Cardiovascular Drugs In Biological Samlples: Calcium channel blocking agent in urine and serum.}

This method has been developed for Verapamil (RamblaAlegre et al., 2006) in serum and urine samples without any pretreatment, by spectrofluorometric detection at $230 \mathrm{~nm}$ (excitation) and $312 \mathrm{~nm}$ (emission). This method is accurate, sensitive and precise. Verapamil is a calcium-channel-blocker. It is basic in nature having $\log \mathrm{K}$ value equal to 9.1. It exists in equilibrium between two forms, one that is molecular form and another that is positively charged. At normal $\mathrm{pH}$ working range of C18 columns i. e. 3-7, the positively charged form of verapamil remains predominant. Additionally Verapamil is a highly hydrophobic compound which leads to inappropriate retention of pure micellar mobile phases of SDS. The mobile phase decided to 
use was $0.15 \mathrm{M}$ SDS and $5 \%$ pentanol at $\mathrm{pH} 7$. The drug was quantified in an adequate analysis time (12.5 min). There was no interference of the protein band or any other endogenous compounds. Calibration was studied in the range of 100-2000 $\mathrm{ng} / \mathrm{ml}$ in three different matrices (micellar solution, serum, and urine), with a regression coefficient of $r>0.998$. Recoveries obtained in biological matrices were $97-99 \%$. This procedure suits in the field of clinical analysis and toxicology.

\section{Determination of beta-antagonists in urine samples.}

Many beta-antagonists (acebutolol, atenolol, celiprolol, labetalol, metoprolol, nadolol, propranolol) were determined with a micellar mobile phase of $0.1 \mathrm{M}$ sodium dodecyl sulfate (SDS), $15 \%$ propanol, and $1 \%$ triethylamine at $\mathrm{pH} 3$ in urine samples with fluorometric detection (Rapado Martínez et al., 1999). The hybrid mobile phase at reduced $\mathrm{pH}$ improved the peak efficiency. The composition of the mobile phase was decided after interpretive procedure. The urine samples were collected from healthy volunteers.

\section{RECENT ADVANCES}

MLC was pioneered by works of Armstrong more than 25 years ago. Since many advances have been taking place. We have focused here some recent and important advancement. $\mathrm{N}$. Memon et al.,. in 2012 studied a nonionic surfactant Brij-35 for its selectivity in the separation of positional isomers. There is different way of interaction of nonionic surfactant as compared with ionic surfactant. The study shows dipolarizability, excess molar refraction and basicity. Such parameters are responsible for separation in case of non ionic MLC. Nonionic MLC has shown a new way for separation of many positional isomers (Najma et al., 2002).

In another case, A. U. Kulikov developed and validated a MLC method for the analysis of sesquiterpenic acids separated from the root and rhizome of Valeriana officinalis without using gradient elution technique. For the HPLC development of same sesquiterpenic acids it is mandatory to use gradient elution technique. This is a very important advantage of MLC which allows separation of compounds having different hydrophobicity in a single run without the gradient elution (Elisa et al., 2002). Another interesting advantage of MLC technique is that direct injection of samples without tedious extraction. M.-L. Chin-Chen determined the biogenic amine spermine in anchovy sauce after derivatizing it with 3, 5-dinitrobenzoyl chloride. Direct injection of samples (after filtration) was used to avoid any extraction and purification step (Rapado et al., 1999).

\section{CONCLUSION}

The success of MLC lies mainly in micellar mobile phase. Various kinds of interactions occur in MLC. Such interactions do not exist in any other mobile phase containing aqueous or organic solvent. Micellar mobile phases are well-suited with a variety of solutes like proteins, blood samples, urine samples, serum samples etc. Direct injection of sample and time saving nature are the very interesting properties of MLC. Hence it has proved as a milestone in the field of bioanalysis.

\section{ACKNOWLEDGEMENT}

Authors overwhelmingly acknowledge the services provided by our mentor, School of Pharmacy, SRTMU, Nanded. Moreover I am deeply indebted to Dr. S. G. Gattani for his keen interest, all time moral support and suggestions in further improvement of my work.

\section{REFERENCES}

Armstrong DW, Nome F. Partitioning behavior of solutes eluted with micellar mobile phases in liquid chromatography. Anal. Chem., 1981;53:1662-1666.

Arunyanart M, Cline-Love LJ. Model for micellar effects on liquid chromatography capacity factors and for determination of micellesolute equilibrium constants.Anal. Chem., 1984; 56 :1557-1561.

Berthod A,Girard I, Gonnet C.Additive effects on surfactant adsorption and ionic solute retention in micellar liquid chromatography. Analytical Chemistry. 1986; 58(7):1362-1367.

Beyer K, Leine D , Blume A. The demicellization of alkyltrimethylammonium bromides in $0.1 \mathrm{M}$ sodium chloride solution studied by isothermal titration calorimetry. Colloids and Surfaces B: Biointerfaces. 2006; 49(1): 31-39.

Borgerding MF , Hinze WL. Characterization and evaluation of the use of nonionic polyoxyethylene(23)dodecanol micellar mobile phases in reversed-phase high-performance liquid chromatography. Analytical Chemistry.1985; 57(12):2183-2190.

Borgerding MF, Hinze WL, Stafford LD, Fulp GW, Hamlin WC. Investigations of stationary phase modification by the mobile phase surfactant in micellar liquid chromatography. Analytical Chemistry. 1989; 61(13): 1353-1358.

Bryant SM, Altria KD. An initial assessment of the use of gradient elution in microemulsion and micellar liquid chromatography. Journal of Separation Science. 2004; 27(17-18):1498-1502.

Capella-Peiro ME, Bose D, Gil-Agusti M, Esteve-Romero J, Carda- Broch S. Direct injection determination of benzoylecgonine, heroin, 6-monoacetylmorphine and morphine in serum by MLC. J. Chromatogr. A. 2005; 1073(1-2): 277-283.

Carda-Broch,S, Gil-Agusti MT, Monferrer-PL, Esteve-Romero JS. Determination of trazodone in urine and pharmaceuticals using micellar liquid chromatography with fluorescence detection. J. Chromatogr. A, 2007; 1156(1-2): 254-258.

Chin-Chen ML, Rambla-Alegre M, Carda-Broch S, EsteveRomero J, Juan Peris-Vicente. 2012. Micellar Liquid Chromatography Determination of Spermine in Fish Sauce after derivatization with 3,5Dinitrobenzoyl Chloride In:Carda-Broch $\mathrm{S}$ ed. Micellar Liquid Chromatography: Recent Advances and Applications. Hindawi publishing corporation.

Delgado B, Pino V, Ayala JH, Gonzalez V, and Afonso AM.Nonionic surfactant mixtures: a new cloud-point extraction approach for the determination of PAHs in seawater using HPLC with fluorimetric detection. Analytica Chimica Acta. 2004;518(1-2) :165-172.

Elisa Capella-Peiro M, Gil-Agusti M, Martinavarro-Domínguez A, Esteve-Romero Determination in serum of some barbiturates using micellar liquid chromatography with direct injection. Anal Biochem. 2002; 309(2): 261-268.

Esteve-Romero J, Carda-Broch S, Gil-Agusti M, Capella-Peiro ME, Bose D. Micellar liquid chromatography for the determination of drug materials in pharmaceutical preparations and biological samples. Trends Anal. Chem. 2005; 24(2): 75-91.

Foley JP. Critical Compilation of Solute-Micelle Binding Constants and Related Parameters from Micellar Liquid Chromatographic Measurements. Anal. Chim. Acta, 1990; 231:237-247. 
Garcia-alvarez-Coque MC, Torres-Lapasio JR, Baeza-Baeza JJ.Modelling of retention behaviour of solutes in micellar liquid chromatography. J. Chromatogr. A.,1997;780:129-148.

Galgano PD, El Seoud OA.Micellar properties of surface active ionic liquids: a comparison of 1-hexadecyl-3-methylimidazolium chloride with structurally related cationic Surfactants.Journal of Colloid and Interface Science. 2010; 345(1):1-11.

Gil-Agusti, M, Carda-Broch S, Capella-Peiro ME, EsteveRomero J. Micellar liquid chromatographic determination of five antianginals in pharmaceuticals. J. Pharm. Biomed. Anal. 2006; 41(4): $1235-1242$

Gil-Agusti M, Carda-Broch S, Monferrer-Pons L, EsteveRomero J, Simultaneous determination of tyramine and tryptamine and their precursor amino acids by micellar liquid chromatography and pulsed amperometric detection in wines. J. Chromatogr. A. 2007;1156(1-2): 288295.

Gil-Agusti M, Esteve-Romero J, Carda-Broch S. Anserine and Carnosine determination in meat samples by pure micellar liquid chromatography. J Chromatogr A. 2008;1189(1-2):444-50.

Heranadez MJM, Alvarez-Coque MCG.Solute-mobile phase and solute-stationary phase interactions in micellar liquid chromatography. A review. Analyst,1992;117:831-837.

Hoonka S, Bose D, Esteve-Romero J, Durgbanshi A. Micellar Liquid Chromatography for the Determination of Some Less Prescribed Benzodiazepines. E- Journal of Chemistry. 2012; 9(1):443450 .

Khaledi MG. Micelles as separation media in high-performance liquid chromatography and high-performance capillary electrophoresis: overview and perspective. J. Chromatogr. A, 1997 ;780 (1 + 2): 3-40.

Kulikov AU. 2012. Micellar LC Separation of Sesquiterpenic Acids and Their Determination in Valeriana officinalis L.Root and Extracts In: Carda-Broch S ed. Micellar Liquid Chromatography:Recent Advances and Applications. Hindawi publishing corporation.

Kulikov AU, Verushkin AG, Loginova LP. Comparison of micellar and reversed-phase liquid chromatography for determination of sulfamethoxazole and trimethoprim. Chromatographia, 2005; 61(9/10):455- 463.

Lavine BK, Hendayana S, He Y, Cooper WT. Solidstate NMR studies of ionic surfactants adsorbed on cyanopropyl bonded phases: implications for micellar liquid chromatography. Journal of Colloid and Interface Science.1996; 179( 2):341-349 .

Lopez-Grio S, Baeza-Bacza JJ, Garcia-Alvarez- Coque MC. Influence of the addition of modifiers on solute micelle interaction in hybrid micellar liquid chromatography.Chromatographia, 1998; 48 (910):655-663.

Lopez-Grio S, Garcia-Alvarez-Coque MC,Hinze WL, Quina $\mathrm{FH}$, Berthod A. Effect of a variety of organic additives on retention and efficiency in micellar liquid chromatography. Analytical Chemistry. 20007; 2(20):4826-4835. 1996;53: 215.

Marina ML Jimenez O, Garcia MA, Vera S. Microchem. J.

Martinez-Algaba C, Bermudez-Saldana JM, VillanuevaCamanas RM, Sagrado S, Medina-Hernandez MJ. Analysis of pharmaceutical preparations containing antihistamine drugs by micellar liquid chromatography. J. Pharm. Biomed. Anal. 2006; 40(2): 312-321.

Mei-Liang Chin-Chen, Bose D, Esteve-Romero J, Peris-Vicente J, Maria Rambl-Alegre Samuel Carda-Broch. Determination of Putrescine and Tyramine in Fish by Micellar Liquid Chromatography with UV Detection Using Direct Injection. The Open Analytical Chemistry Journal. 2011; 5: 22-26

Mittal KL, Ed., Micellization. Solubilization and Microemulsions, vol. 1, Plenum Press, New York, NY, USA, 1979.

Murray RC, Hartley GS. Equilibrium between micelles and simple ions, with particular reference to the solubility of long-chain salts. Transactions of the Faraday Society. 1935;31:183-189.
Myers D. 1988. Surfactant Science and Technology. New York,USA: VCH Publishers.

Najma M, Huma IS, Amber RS. 2012.Selectivity of Brij-35 in Micellar Liquid Chromatographic Separation of Positional Isomers. In: Carda-Broch S ed. Micellar Liquid Chromatography:Recent Advances and Applications. Hindawi publishing corporation.

Nishi H. Pharmaceutical applications of micelles in chromatography and electrophoresis. J. Chromatogr. A. 1997; 780(1-2): 243-264.

Pino V, Yao C, Anderson JL. Micellization and interfacial behavior of imidazolium-based ionic liquids in organic solvent-water mixtures. Journal of Colloid and Interface Science. 2009;33(32):548-556.

Rambla-Alegre M. 2012. Basic Principles of MLC. In : CardaBroch S ed. Micellar Liquid Chromatography:Recent Advances and Applications. Hindawi publishing corporation 1-6.

Rambla-Alegre M, Gil-Agusti MT, Capella-Peiro ME, CardaBroch S, Esteve-Romero JS, Direct determination of verapamil in urine and serum samples by micellar liquid chromatography and fluorescence detection. J. Chromatogr. B.2006; 839(1-2): 89-94.

Rapado M, Villanueva Camanas RM, Garcia Alvarez-Coque MC. Micellar liquid chromatography: A worthy technique for the determination of beta-antagonists in urine samples. Anal Chem. 1999;71(2):319-326.

Rosen MJ 2004. Surfactants and interfacial phenomena. New Jersey, USA: Wiley.

Ruiz-Angel MJ, Caballero RD,. Sim-o-Alfonso EF, GarciaAlvarez-Coque MC.Micellar liquid chromatography: suitable technique for screening analysis. Journal of Chromatography A. 2002; 947(1):31-45.

Ruiz-Angel MJ, Carda-Brochb S, Torres-Lapasioa JR, M.C. Garcia-alvarez-Coquea Retention mechanisms in micellar liquid chromatography, Journal of Chromatography A, 2009;1216: 1798-1814.

Ruiz-Angel MJ, Torres-Lapasio JR, Garcia-Alvarez- Coque MC, Carda-Broch S. Retention mechanisms for basic drugs in the submicellar and micellar reversed-phase liquid chromatographic modes. Analytical Chemistry, 2008; 80(24):9705-9713.

Ruiz-Angel MJ, Torres-Lapasio JR, Garcia-Alvarez- Coque MC, Carda-Broch S. Submicellar and micellar reversed- phase liquid chromatographic modes applied to the separation of $\beta$-blockers. Journal of Chromatography A, 2009; 1216 (15): 3199-3209.

Sehgal P , Otzen DE. Thermodynamics of unfolding of an integral membrane protein in mixed micelles. Protein Science. 2006;15(4):890-899.

Srisom P, Liawruangrath B, Liawruangrath S. Micellar liquid chromatographic determination of penicillins in pharmaceuticals. Chromatographia. 2007; 65(11/12): 687- 693.

Texter J. 1999. Characterization of Surfactants, in Surfactants, A Practical Handbook. In : Lange KR , ed. Hanser Gardner Publications, Inc: Cincinnati.1-68.

Thomas DP. Efficiency enhancements in micellar liquid chromatography through selection of stationary phase and alcohol modifier. journal of chromatography A, 2007; 1149(2), 282-293.

Torres-Cartas S, Martin-Biosca Y, Sagrado S, VillanuevaCamanas RM, Medina Hernandez MJ. Comparison between micellar liquid chromatography and capillary zone electrophoresis for the determination of hydrophobic basic drugs in pharmaceutical preparations. Biomed. Chromatogr. 2007; 21(1): 21-28.

\section{How to cite this article:}

Kalyankar T. M., Kulkarni P.D., Wadher S. J. and Pekamwar S. S. Applications of Micellar Liquid Chromatography in Bioanalysis: A Review. J App Pharm Sci, 2014; 4 (01): 128-134. 